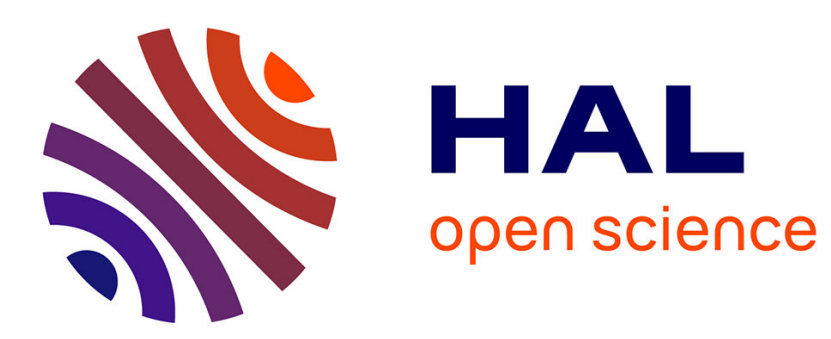

\title{
Variational multi-wavelet restoration of noisy images
}

Ronan Fablet, Jean-Marie Augustin

\section{To cite this version:}

Ronan Fablet, Jean-Marie Augustin. Variational multi-wavelet restoration of noisy images. ICIP 2005 : IEEE International Conference on Image Processing, Sep 2005, Genova, Italy. pp.305 - 308, 10.1109/ICIP.2005.1530389 . hal-02344402

\section{HAL Id: hal-02344402 \\ https://hal.science/hal-02344402}

Submitted on 4 Nov 2019

HAL is a multi-disciplinary open access archive for the deposit and dissemination of scientific research documents, whether they are published or not. The documents may come from teaching and research institutions in France or abroad, or from public or private research centers.
L'archive ouverte pluridisciplinaire HAL, est destinée au dépôt et à la diffusion de documents scientifiques de niveau recherche, publiés ou non, émanant des établissements d'enseignement et de recherche français ou étrangers, des laboratoires publics ou privés. 


\title{
VARIATIONAL MULTI-WAVELET RESTORATION OF NOISY IMAGES
}

\author{
Ronan Fablet, Jean-Marie Augustin \\ IFREMER/TMSI/AS \\ BP 70, 29280 Plouzane,France \\ \{rfablet,augustin\}@ifremer.fr
}

\begin{abstract}
This paper presents a new technique for noise removal in images. It benefits both from the recent advances in waveletbased and variational denoising. Whereas wavelet-based analysis tends to strongly depend on the selected wavelet basis, we propose to combine and fuse several mono-wavelet analysis within a variational framework. The associated energy function involves M-estimator in order to guarantee the robustness to outliers and to preserve image structures (edges, ridges,...). An experimental evaluation for a Gaussian additive noise validates the proposed approach and an application to speckle removal in sonar sea-bed images highlights the interest of this approach for real images.
\end{abstract}

\section{PROBLEM STATEMENT AND RELATED WORK}

Noise removal in images is a common issue for a wide range of applications: multimedia, teledetection, underwater imaging, medical imaging,.... Two main categories of techniques have been recently proposed to cope with image restoration. On the one hand, wavelet-based approaches $[1,6,9,10]$ cope with this issue through the shrinkage of wavelet coefficients. Despite good performances, these approaches tend to produce local artifacts for high level noise. On the other hand, variational methods [7, 8] based on non-quadratic criteria (e.g., the total variation) have been introduced to state image denoising as a minimization issue. This energy-based setting usually involves two terms: a regularity term defined on the image gradient and a data-driven term measuring the distance between the noisy image and the denoised one.

Therefore, it seems appealing to benefit from both categories of approaches. For instance, in [2], wavelet shrinkage was stated in a variational setting through the minimization of a total variation criterion. In this paper, we give the emphasis to a multi-wavelet approach while stating the image restoration within a variational framework. Since waveletbased denoising performances are known to strongly depend on the chosen wavelet basis, we aim at combining several wavelet analysis. To this end, the proposed variational setting provides a well-formalized fusion criterion. The subsequent is organized as follows. In Section 2, we briefly review mono-wavelet image denoising. The proposed variational multi-wavelet approach is described in Section 3. Section 4 present experimental results on synthetic images and an application to speckle removal in underwater sonar images.

\section{WAVELET SHRINKAGE}

Wavelet shrinkage for noise removal was first introduced by Donoho etal [1]. The detail coefficients of a wavelet decomposition are indicators of the presence of signal ruptures: the greater the more relevant for image reconstruction. Consequently, the restoration of noisy images can be viewed as the shrinkage of these wavelet coefficients. Initially, Donoho etal. [1] have proposed the hard- and softshrinkage schemes. For instance, given an image $I$ and the associated wavelet decomposition $W I$, the hard-shrinkage of the detail coefficients $\left\{W I_{k, l}\right\}$, where $k$ refers to the scale level and $l$ to the type of detail coefficients (horizontal, vertical or diagonal), resorts to:

$$
\forall p, \widetilde{W I}_{k, l}(p)=\left\{\begin{array}{cl}
0, & \text { if } W I_{k, l}(p)<\delta \\
W I_{k, l}(p), & \text { otherwise }
\end{array}\right.
$$

The threshold $\delta$ is set w.r.t. the noise characteristics: for instance, for a Gaussian additive noise of standard deviation $\sigma, \delta$ is typically set to $3 \sigma$. The filtered image $\tilde{I}$ simply results from the reconstruction using the modified wavelet coefficients $\widetilde{W I}$ :

$$
\tilde{I}=\widetilde{W I}_{N, 0} \Phi_{N, 0}+\sum_{k=1}^{N} \sum_{l=1}^{3} \widetilde{W I}_{k, l} \Phi_{k, l}
$$

where $\Phi_{k, l}$ is the wavelet basis function at resolution $k$ for the quarter $l$ ( $l=0$, low frequency, $l=1,2,3$; horizontal, vertical and diagonal high frequency).

The shrinkage rules can also be deduced from a Bayesian setting $[6,9]$ when a priori information on the distribution of the wavelet coefficients is considered. For instance, in [9], a bivariate shrinkage scheme exploiting the inter-scale 
dependencies is proposed:

$$
\forall p, \widetilde{W I}_{k, l}(p)=\frac{\left(\alpha(k, l, p)-\sqrt{3} \sigma_{N} / \sigma\right)_{+}}{\alpha(k, l, p)} W I_{k, l}(p)
$$

with $\alpha(k, l, p)=\sqrt{W I_{k, l}^{2}(p)+W I_{k+1, l}^{2}\left(p^{+}\right)}$, where $p^{+}$is the father of the point $p$ in the wavelet decomposition. $\sigma_{N}$ is the standard deviation of the Gaussian noise and $\sigma$ the deviation of the a priori model for inter-scale dependencies. This soft shrinkage scheme was proven experimentally to outperform Donoho's scheme [9]. In the subsequent, we will use this scheme to perform a multi-wavelet analysis.

\section{VARIATIONAL MULTI-WAVELET DENOISING}

The key contribution of this paper is to introduce a wellformalized framework to achieve a multi-wavelet denoising. As illustrated in Fig.1, the performance of waveletbased denoising techniques strongly depend on the choice of the wavelet basis. Besides, whereas a variety of wavelet basis (e.g., daubechies, curvelets, ridgelets,...) has been introduced $[5,11]$, real images usually involve different types of structures. Hence, selecting only one wavelet basis might not be optimal.

Rather than selecting an optimal wavelet basis, we propose to exploit the decompositions obtained from several wavelet basis. Image restoration then comes to fuse these analysis within a variational framework detailed below.

\subsection{Robust energy setting}

Given a noisy image $I$ and a chosen set of wavelet basis $\mathcal{B}$, we first apply the bivariate shrinkage for each wavelet basis in $\mathcal{B}$ and compute the resulting filtered outputs $\left\{\tilde{I}^{b}\right\}_{b \in \mathcal{B}}$. The fusion step is then stated as the following variational issue:

$$
\min \int \sum_{b \in \mathcal{B}} \rho_{b}\left(\tilde{I}(p)-\tilde{I}^{b}(p)\right)+\int \rho_{2}(\nabla I(p))
$$

where $\rho_{i}$ a M-estimator (e.g., Welsch M-estimator $\rho(x)=$ $1-\exp \left(-x^{2} / \sigma^{2}\right)$ with $\sigma$ a scale parameter $)$ and $\gamma$ a weight balancing the importance of the two energy terms. The first term can be referred to as the data-driven term: it states that the restored image $\tilde{I}$ should be close to the mono-wavelet result $\left\{\tilde{I}^{b}\right\}$. The use of the M-estimator $\rho_{1}$ guarantees the robustness to outliers such as local artifacts. The second term set on the image gradient is aimed at imposing some regularity constraints on the output image. Whereas quadratic criteria are known to oversmooth meaningful image structures (edges, ridges,...), the M-estimator $\rho_{2}$ provides a mean to preserve these structures in the filtered image, while smoothing uniform areas.
The proposed variational setting can be regarded as a way to drive the fusion of several mono-wavelet analysis by a regularity constraint.

\subsection{Minimization issue}

Rather than exploiting the Euler-Lagrange partial derivative equation derived from Eq.4, we adopt a discrete Markovian setting. The energy in minimization (4) then resorts to:

$$
\begin{aligned}
J(I) & =\sum_{p} \sum_{b \in \mathcal{B}} \rho_{b}\left(I(p)-\tilde{I}^{b}(p)\right) \\
& +\sum_{(p, q) \in \mathcal{V}} \rho_{2}(I(p)-I(q))
\end{aligned}
$$

where $\in \mathcal{V}$ is the 4-neighborhood.

The minimization of function $J(I)$ is then solved for using an iterated reweighted least-square scheme [4]. It iterates two steps. First, given the current estimate $\tilde{I}$, the following robust weights are computed:

$$
\begin{gathered}
\forall p, \forall b \in \mathcal{B}, \quad w_{b}(p)=\psi_{1}\left(\tilde{I}(p)-\tilde{I}^{b}(p)\right) \\
\forall(p, q) \in \mathcal{V}, \quad w_{2}(p, q)=\psi_{2}(\tilde{I}(p)-\tilde{I}(q))
\end{gathered}
$$

where $\psi_{i}$ is the influence function associated to $\rho_{i}$ (i.e., $\left.\psi_{i}(r)=1 / r \cdot d \rho / d r(r)\right)$. The second step comes to minimize the reweighted least-square function $\tilde{J}(I)$ given by:

$$
\begin{aligned}
J(I) & =\sum_{p} \sum_{b \in \mathcal{B}} w_{1}(p)\left\|I(p)-\tilde{I}^{b}(p)\right\|^{2} \\
& +\sum_{(p, q) \in \mathcal{V}} w_{2}(p, q)\|I(p)-I(q)\|^{2}
\end{aligned}
$$

This minimization is carried out using the ICM algorithm. The initialization is provided by the mean over the monowavelet outputs:

$$
\tilde{I}_{0}=\frac{1}{|\mathcal{B}|} \sum_{b \in \mathcal{B}} \tilde{I}^{b}
$$

In practice, we use Welsch M-estimator $\rho_{i}(x)=1-\exp \left(-x^{2} / \sigma_{i}^{2}\right)$. The scale parameters $\left(\left\{\sigma_{b}\right\}, \sigma_{2}\right)$ are initially set according to the median value of the residuals:

$$
\begin{gathered}
\sigma_{b}=1.57 \operatorname{med}\left(\left|\tilde{I}(p)-\tilde{I}^{b}(p)\right|\right) \\
\sigma_{2}=1.57 \operatorname{med}[|\tilde{I}(p)-\tilde{I}(q)|,(p, q) \in \mathcal{V}]
\end{gathered}
$$

and then updated w.r.t. a geometrical law at each iteration. 


\subsection{Coarse-to-fine strategy}

In order to improve the convergence of the proposed scheme, we take advantage from the multiresolution structure of the wavelet decompositions to design a coarse-to-fine strategy.

At a given level resolution $k^{*}$, we first reconstruct the mono-wavelet outputs $\left\{\tilde{I}_{k^{*}}^{b}\right\}_{b \in \mathcal{B}}$ from the shrinked wavelet coefficients $\left\{\widetilde{W I}^{b}\right\}_{b \in \mathcal{B}}$ using only resolution levels greater than $k^{*}$ :

$$
\tilde{I}_{k^{*}}^{b}=\widetilde{W I^{b}}{ }_{N, 0} \Phi_{N, 0}+\sum_{k=k^{*}}^{N} \sum_{l=1}^{3} \widetilde{W I}_{k, l}^{b} \Phi_{k, l}
$$

Minimization (4) is carried out w.r.t. $\left\{\tilde{I}_{k}^{b}\right\}_{b \in \mathcal{B}}$ to determine the filtered image $\tilde{I}_{k^{*}}$ at resolution $k^{*}$. Given $\tilde{I}_{k^{*}}$, we compute the associated wavelet decompositions $\left\{W \tilde{I}_{k^{*}}^{b}\right\}_{b \in \mathcal{B}}$, which are used to update the wavelet decompositions $\left\{\widehat{W I}^{b}\right\}_{b \in \mathcal{B}}$ at level $k^{*} k$ :

$$
\forall b, \forall l, \widetilde{W I}_{k^{*}, l}^{b}=\left(W \tilde{I}_{k^{*}}^{b}\right)_{k^{*}, l}
$$

This procedure is iterated at resolution $k^{*}-1$ using the updated wavelet decompositions $\left\{\widetilde{W I}^{b}\right\}_{b \in \mathcal{B}}$. The output at the finest resolution provides the filtered image.

\section{EXPERIMENTS}

\subsection{Quantitative analysis on synthetic images}

To validate the proposed approach, different experiments have been carried out both for synthetic and real images. In both cases, we use the same parameter setting: wavelet decomposition with 4 levels, a set of 10 wavelet basis (haar, dauchechies 4, 6 and 8, coiflets 1, 2 and 3, and symlets 4, 5 and 6$), \gamma=10$. In a first step, we have performed a quantitative analysis for two synthetic images, reported in Fig.1, w.r.t. to different an additive Gaussian noise with a variance from 10 to 90 . It demonstrates that, in any case, the multi-wavelet denoising outperforms all the mono-wavelet ones. In addition, the results also stress the improvements brought by combining all mono-wavelet filtering though the proposed variational fusion scheme. The average gain resulting from the fusion scheme is of $0.75 \mathrm{~dB}$ w.r.t. the best mono-wavelet denoising and $2 \mathrm{~dB}$ w.r.t. the worst one.

\subsection{Application to speckle removal in seabed sonar im- ages}

We apply the proposed multi-wavelet variational denoising to speckle removal in seabed sonar images. Fig.3 depicts an example of sonar image. Such images are highly corrupted by a multiplicative speckle noise.

In order to resort to an additive noise, we first process the logarithm of the sonar image. The resulting noise can be

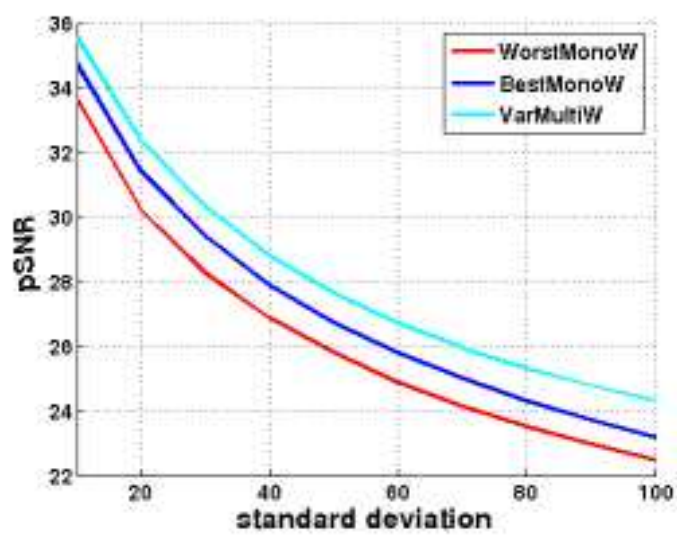

Fig. 1. Comparison of the denoising results for an additive Gaussian with a variance from 10 to 100 applied to "lena" $\therefore$ we plot the evolution of the pSNR as a function of the standard deviation of the additive Gaussian noise for the worst (WorstMonoW) and best (BestMonoW) mono-wavelet denoising result and the result of the variational multi-wavelet method (VarMultiW).

roughly approximated as a Gaussian noise. We then apply the proposed denoising scheme and reconstruct the filtered image using the exponential function.

Fig. 3 reports an example of speckle removal for a seabed sonar image depicting a wreck lying on sand. We provide a comparison of the proposed multi-wavelet variational denoising and of the Frost scheme [3]. The Frost scheme is a locally adaptive linear filter. The zoom on details of the wreck clearly stress the improvements brought by our approach. Local details such as masts are better separated from noise using the variational approach.

\section{CONCLUSION AND FUTURE WORK}

In this paper, we have coped with the restoration of noisy images. We have emphasized the potential improvements brought by combining several wavelet analysis w.r.t. different types of wavelet basis or different parametrization of a given wavelet basis. This fusion issue is stated in a robust variational framework. The use of robust estimators allow us to cope with the presence of outliers in the mono-wavelet denoising outputs and to preserve relevant image structures such as edges. The experiments carried out for synthetic images corrupted by an additive Gaussian noise quantitatively validate the improvement brought by our scheme compared to mono-wavelet schemes. Besides, the application to speckle removal in sea-bed sonar images demonstrate its efficiency to cope with image restoration in complex real cases.

In future work, we will investigate the adaption of our 
noisy image

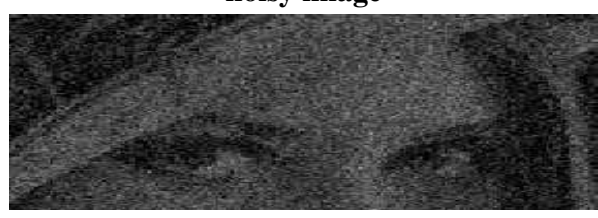

Best mono-wavelet denoising

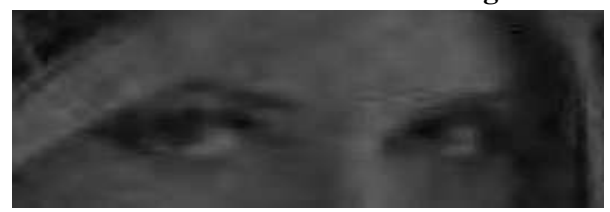

multi-wavelet variational denoising

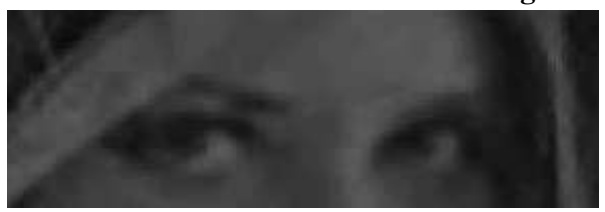

Fig. 2. Comparison of the multi-wavelet variational denoising to the best mono-wavelet denoising for "lena" corrupted by a Gassian additive noise with a standard deviation set to 40. We display a zoom on the area around the eyes.
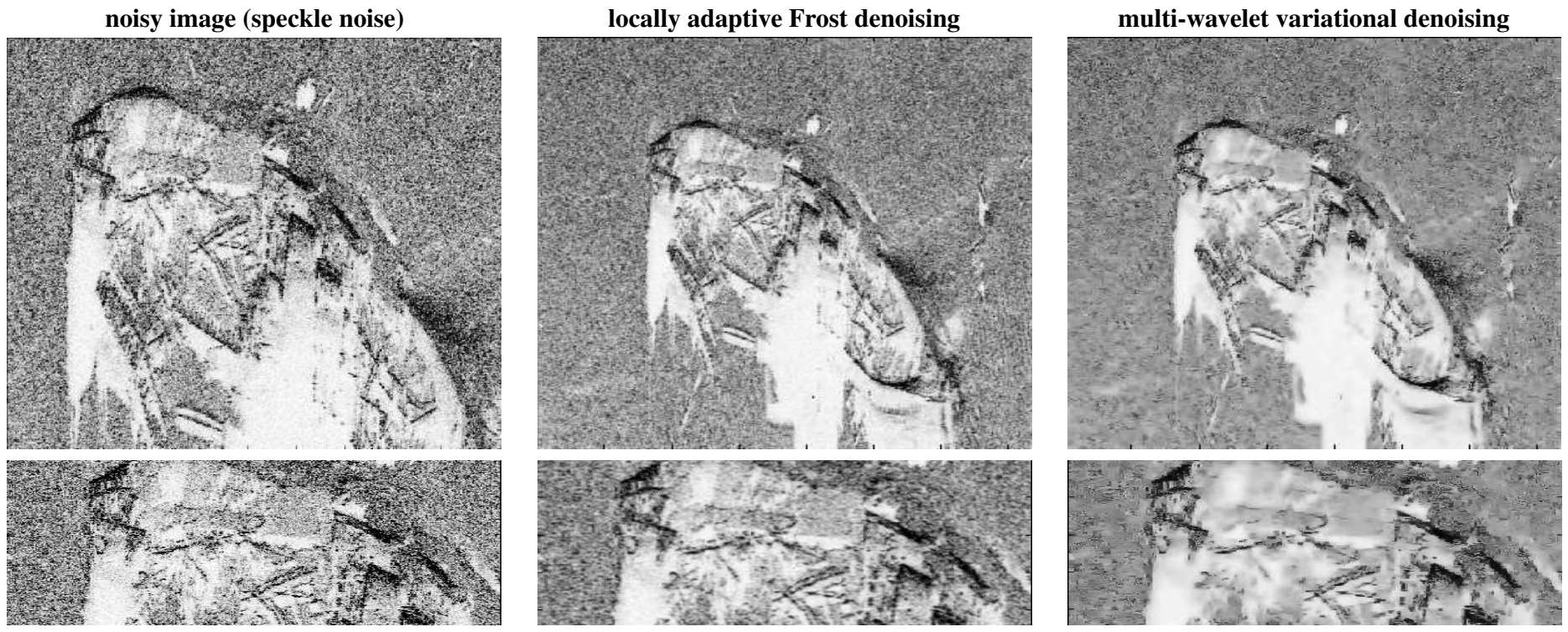

Fig. 3. Speckle removal for the sea-bed sonar image "Swansea" (we are thankful to GESMA for providing this image): comparison of the proposed multi-wavelet variational method to the locally adaptive Frost technique [3].

variational multi-wavelet scheme to local noise characteristics. Besides, concerning the application to the analysis of sonar images, it might be relevant to better account for the characteristics of shadow areas.

\section{REFERENCES}

[1] D. Donoho. De-noising by soft-thresholding. IEEE Trans. on Information Theory, 41(3):613-627, 1995.

[2] S. Durand and J. Froment. Reconstruction of wavelet coefficients using total variation minimization. SIAM Jal Scientific Computing, 24(5):1754-1767, 2003.

[3] V.S. Frost, J.A. Stiles, K.S. Shanmugan, and J.C. Holtzman. A model for radar images and its application to adaptive digital filtering of multiplicative noise. IEEE Trans. on PAMI, 4:157-165, 1982.

[4] P. Huber. Robust Statistics. John Wiley \& Sons, 1981.

[5] S. Mallat. A wavelet tour of signal processing. Academic Press, 1998.

[6] A. Pizurica, W. Philips, I. Lemahieu, and M. Acheroy. A joint inter- and intrascale statistical model for bayesian wavelet based image denoising. IEEE Trans. on Image Processing, 11(5):545-557, 2002.

[7] L. Rudin, S. Osher, and E. Fatemi. Nonlinear total variation based noise removal algorithms. Physica D, 60(1-4):259_ 268, 1992.

[8] C. Samson, L. Blanc-Féraud, G. Aubert, and J. Zerubia. A variational model for image classification and restoration. IEEE Trans. on PAMI, 22(5):460-472, 2000.

[9] L. Sendur and I.W. Selesnick. Bivariate shrinkage functions for wavelet-based image denoising. ieee-sp, 50(11):2744 2756, 2002.

[10] C. Sintes, M. Legris, and B. Solaiman. Interferometric side scan sonar signal denoised by wavelets. In Trans. of SPIE AeroSense Int. Symp. on Aerospace/Defense Sensing, Simulation and Controls, Orlando, Apr. 2003.

[11] J.L. Starck, E. Candes, and D.L. Donoho. The curvelet transform for image denoising. IEEE Trans. on Image Processing, 11(6):670-684, 2002. 